\title{
Current practice of diagnosis and reporting of prostatic intraepithelial neoplasia and glandular atypia among genitourinary pathologists
}

\author{
Lars Egevad ${ }^{1}$, William C Allsbrook $\mathrm{Jr}^{2}$ and Jonathan I Epstein ${ }^{3}$ \\ ${ }^{1}$ Department of Pathology and Cytology, Karolinska Hospital, Stockholm, Sweden; ${ }^{2}$ Departments of Pathology \\ and Surgery (Urology), Medical College of Georgia, Augusta, USA and ${ }^{3}$ Departments of Pathology, Oncology \\ and Urology, Johns Hopkins Hospital, Baltimore, USA
}

\begin{abstract}
The criteria for diagnosing prostatic intraepithelial neoplasia (PIN) and lesions suspicious for cancer are described in the literature. However, it is unknown how these are applied in practice by experts in genitourinary (GU) pathology. A questionnaire was sent to $93 \mathrm{GU}$ pathologists in countries around the world with the purpose of surveying current practices. The response rate was $69 \%$ including $\mathbf{4 0}$ North American pathologists and 24 from other continents. For preneoplastic lesions, the term PIN was universally endorsed by the respondents. PIN was graded by $83 \%$, usually as low/high-grade PIN (LGPIN/HGPIN) or as HGPIN only. Most respondents would usually not report lesions that may qualify for LGPIN. A majority (81\%) did not specify architectural patterns of PIN. With both HGPIN and invasive cancer present, $69 \%$ would still mention HGPIN. Among the diagnostic criteria for HGPIN were any nucleoli visible (52\%), or nucleoli seen in at least $10 \%$ of cells (33\%). However, $56 \%$ would diagnose HGPIN in the absence of prominent nucleoli, most commonly based on prominent pleomorphism, marked hyperchromasia or mitotic figures. The number of cores involved with HGPIN was specified by $\mathbf{5 0} \%$. Lesions suspicious for but not diagnostic of carcinoma were reported by $45 \%$ as atypia, atypical glands or suspicious for cancer and by $42 \%$ as atypical small acinar proliferation. The degree of suspicion was further defined by $41 \%$. Our survey data may serve as a guideline to general pathologists on how to diagnose and report atypia and PIN in prostate biopsies.
\end{abstract}

Modern Pathology (2006) 19, 180-185. doi:10.1038/modpathol.3800522; published online 2 December 2005

Keywords: prostatic neoplasms; prostatic intraepithelial neoplasia; pathology; male; human

We have recently surveyed current practices of Gleason grading of prostate cancer among experts in genitourinary (GU) pathology. ${ }^{1}$ On some issues, there was a high level of consensus among respondents, while on others, there was a significant disagreement, calling for standardization. There are reasons to believe that diagnosis and reporting of prostatic intraepithelial neoplasia (PIN) and lesions suspicious for cancer may also suffer from lack of uniformity. Over the last decades there has been a shift in nomenclature for these diagnoses. Lesions that used to be diagnosed as atypical hyperplasia or dysplasia are now known as PIN. ${ }^{2}$

Correspondence: Dr L Egevad, MD, PhD, Department of Pathology and Cytology, Karolinska Hospital, Radiumhemmet P1:02, Stockholm SE-171 76, Sweden.

E-mail: lars.egevad@onkpat.ki.se

Received 17 August 2005; revised 13 October 2005; accepted 16 October 2005; published online 2 December 2005
For lesions suspicious of cancer, the term atypical small acinar proliferation (ASAP) has been suggested..$^{3,4}$ It is unclear to what extent new terminology has been adopted worldwide. Criteria for diagnosing PIN have also evolved in recent years, and definitions have focused on nucleolar prominence. ${ }^{5}$ Grading of preneoplastic lesions has moved from a three-tier system (PIN 1-3) to a two-tier system (LGPIN and HGPIN). ${ }^{5}$

The purpose of this study was to survey current practice among GU pathologists of diagnosing and reporting PIN and lesions suspicious of but not diagnostic for prostatic carcinoma.

\section{Materials and methods}

A questionnaire was distributed to 93 GU pathologists in countries around the world. Participants were included because they were well known by the 
authors as experts in prostate pathology. Most of them (90) were recruited from a group of pathologists who were invited to a recent survey study on Gleason grading of prostate cancer. ${ }^{1}$ The questionnaire included questions on demographic and professional data (age, nationality and type of practice) and questions on how PIN and glandular atypia are diagnosed and reported. All questions had fixed response alternatives but, in some, the respondents were also allowed to give other alternatives.

\section{Statistical Analysis}

The $\chi^{2}$ test was used for the comparison of proportions when analyzing differences between age categories and nationalities. A $P$-value less than 0.05 was considered significant.

\section{Results}

\section{Demographics}

Of 62 invited North American pathologists, 40 $(65 \%)$ replied, and of 31 invited pathologists from other continents, $24(77 \%)$ replied, corresponding to an overall response rate of $69 \%(64 / 93)$. In all, 40 (63\%) respondents were North American (37 from United States and three from Canada) and 24 (37\%) were from other continents including 15 from Europe, four from Asia, two from South America and three from Australia or New Zealand. Of the 64 pathologists who replied, 43 worked only at an academic institution, seven only in private health care and 10 had a combined academic and private practice. In total, 17 were involved in private health care and among them, 13 worked in a private hospital, five in a private general laboratory and four in a private laboratory primarily devoted to prostatic pathology (more than one alternative possible). Four pathologists worked at other institutions including military or nonacademic community health care. The age categories 31-40, 41-50, 51-60, 61-70 and > 70 years included six $(9 \%), 20$ $(31 \%), 24(28 \%), 13(20 \%)$ and one $(2 \%)$ persons, respectively.

\section{Prostatic Intraepithelial Neoplasia}

All respondents used the designation PIN for preneoplastic lesions of the prostate, while none preferred dysplasia, duct-acinar dysplasia, atypia, atypical hyperplasia, intraductal carcinoma or carcinoma in situ (Table 1).

An intraductal prostatic glandular lesion (verified by basal cell stains) that has necrosis or much more pronounced cytological or architectural atypia than typical PIN would be diagnosed as intraductal carcinoma of the prostate with or without an explanatory comment by 28 (44\%), HGPIN with a
Table 1 Terminology and grading of PIN

\begin{tabular}{|c|c|}
\hline Question & $\begin{array}{c}\text { Percent } \\
\text { (number) of } \\
\text { respondents }\end{array}$ \\
\hline \multicolumn{2}{|l|}{ Terminology of preneoplastic lesions } \\
\hline PIN & $100(64)$ \\
\hline Dysplasia & $0(0)$ \\
\hline Duct-acinar dysplasia & $0(0)$ \\
\hline Atypia & $0(0)$ \\
\hline Atypical hyperplasia & $0(0)$ \\
\hline Intraductal carcinoma & $0(0)$ \\
\hline Carcinoma in situ & $0(0)$ \\
\hline \multicolumn{2}{|c|}{$\begin{array}{l}\text { Terminology of intraductal/glandular lesion with pronounced } \\
\text { cytological or architectural atypia or necrosis }\end{array}$} \\
\hline $\begin{array}{l}\text { Intraductal carcinoma with or without a } \\
\text { comment }\end{array}$ & $44(28)$ \\
\hline HGPIN with a comment on atypical features & $27(17)$ \\
\hline HGPIN without a comment on atypical features & $20(13)$ \\
\hline \multicolumn{2}{|l|}{ Grading of PIN } \\
\hline Yes & $83(53)$ \\
\hline No & $17(11)$ \\
\hline \multicolumn{2}{|l|}{ Method for grading of PIN } \\
\hline Only HGPIN & $59(38)$ \\
\hline LGPIN and HGPIN & $17(11)$ \\
\hline Only PIN 2 and 3 & $8(5)$ \\
\hline PIN 1-3 & $0(0)$ \\
\hline \multicolumn{2}{|l|}{ LGPIN } \\
\hline Never mentioned & $58(37)$ \\
\hline $\begin{array}{l}\text { Never mentioned in diagnosis, but rarely in } \\
\text { descriptive part if prominent }\end{array}$ & $25(16)$ \\
\hline $\begin{array}{l}\text { Never mentioned in diagnosis, but routinely in } \\
\text { descriptive part }\end{array}$ & $11(7)$ \\
\hline Rarely mentioned in diagnosis if prominent & $5(3)$ \\
\hline
\end{tabular}

comment on the more atypical features by 17 (27\%), and HGPIN without such a comment by $13(20 \%)$. A descriptive diagnosis without a specific name was given by six $(9 \%)$.

PIN was graded by 53 (83\%). The categories used for grading of PIN were only HGPIN used by 38 (59\%), LGPIN and HGPIN used by $11(17 \%)$, only PIN 2-3 used by five ( $8 \%$ ) and other categories used by one $(2 \%)$. None of the respondents used PIN 1-3.

For a lesion that might qualify for LGPIN (PIN 1) found on needle biopsy, 37 (58\%) would never mention it in either the diagnosis or in the descriptive part of the report, $16(25 \%)$ would never mention it in the diagnosis, yet rarely mention it in the descriptive part of the report if the lesion stands out quite prominently, seven $(11 \%)$ would never mention it in the diagnosis, yet routinely mentioned it in the descriptive part of the report and three (5\%) would rarely mention it in the diagnosis in cases where the lesion stands out quite prominently. None of the respondents would routinely mention it in the diagnosis. One $(2 \%)$ would diagnose LGPIN as atypical hyperplasia. If LGPIN is mentioned in any of the above circumstances, $10(16 \%)$ would add a note that it is not clinically significant. 
Architectural patterns of PIN were specified in needle biopsy reports by $12(19 \%)$ (Table 2). They were specified by $13 \%$ of North Americans and by $29 \%$ of others $(P=0.10)$. Patterns specified were cribriform pattern by 10 (16\%), micropapillary pattern by eight $(13 \%)$, tufted pattern by eight $(13 \%)$ and flat pattern by eight $(13 \%)$. No one would give a comment on the significance of the pattern in the report.

When HGPIN but no invasive cancer is found in a needle biopsy, four (6\%) would always add a comment to the report that HGPIN is a premalignant lesion, nine (14\%) would sometimes do so depending on who the referring doctor is, five $(8 \%)$ would

Table 2 Reporting, immunohistochemistry and diagnostic criteria of PIN

\begin{tabular}{lc}
\hline Question & $\begin{array}{c}\text { Percent } \\
\text { (number) of } \\
\text { respondents }\end{array}$ \\
\hline Specification of architectural patterns of PIN & $19(12)$ \\
Yes & $81(52)$ \\
No & \\
Comment that HGPIN without invasive cancer is & premalignant \\
Yes, always & $6(4)$ \\
Sometimes (depending on referring doctor) & $14(9)$ \\
Sometimes (depending on patient age) & $8(5)$ \\
No & $75(48)$
\end{tabular}

Recommendation of repeat biopsy after HGPIN without invasive cancer

Yes, always 8 (5)

Sometimes (depending on referring doctor) 25 (16)

Sometimes (depending on patient age) $13(8)$

No

$63(40)$

Mention HGPIN if concomitant cancer Yes $69(44)$

No $31(20)$

Immunohistochemistry for basal cells on needle biopsy with HGPIN

Never $23(15)$

$<5 \% \quad 44(28)$

$5-19 \%$

$20-49 \% \quad 2(1)$

$50-99 \%$

Always $3(2)$

Diagnose HGPIN without prominent nucleoli Yes

No

$56(36)$

$44(28)$

Prominent nucleoli required for HGPIN

Any visible at $\times 40$

$16(10)$

Any visible at $\times 20$

$16(10)$

$19(12)$

Any visible regardless of magnification

$11(7)$

$9(6)$

In $\geq 10 \%$ of secretory cells at $\times 20$

$13(8)$

In $\geq 10 \%$ of secretory cells regardless of magnification

Reporting of number of cores with HGPIN Yes

No

$50(32)$

$50(32)$ sometimes do so depending on the patient's age and $48(75 \%)$ never would. Repeat biopsies would always be recommended in the report by five $(8 \%)$, while $16(25 \%)$ sometimes would depending on who the referring doctor is, eight $(13 \%)$ would depending on the patient's age, and $40(63 \%)$ never would. In needle biopsy cases with both HGPIN and invasive cancer, $44(69 \%)$ would mention the presence of HGPIN in the report.

When a lesion consistent with HGPIN is found on hematoxylin- and eosin-stained sections of a needle biopsy, an immunohistochemical stain for basal cells was never done by $15(23 \%)$, in less than $5 \%$ of cases by $28(44 \%)$, in $5-19 \%$ by $16(25 \%)$, in $20-49 \%$ by one $(2 \%)$, in $50-99 \%$ by two $(3 \%)$, and always by two (3\%).

HGPIN would be diagnosed in the absence of prominent nucleoli by $36(56 \%)$. This was less common among pathologists older than 50 years $(45 \%)$ than among those younger than that $(73 \%)$, $P=0.02$. To diagnose HGPIN in the absence of prominent nucleoli, one or several of the following features would be required: prominent pleomorphism 34 (53\%), marked hyperchromasia $30(47 \%)$, presence of mitotic figures 18 (28\%), micropapillary pattern seven (11\%), cribriform pattern $12(19 \%)$ or extensive lesion five $(8 \%)$.

HGPIN would be diagnosed by $10(16 \%)$ if prominent nucleoli were seen at $\times 40$ objective lens magnification, by $11(17 \%)$ if they were seen at $\times 20$ magnification and by $12(19 \%)$ if seen regardless of magnification. It was required by seven $(11 \%)$ that prominent nucleoli were found in at least $10 \%$ of the secretory cells of the acinus at $\times 40$ objective lens magnification by six $(9 \%)$ that they were seen in at least $10 \%$ of the secretory cells acinus at $\times 20$ magnification, and eight (13\%) would require that they be seen in $10 \%$ of the secretory cells of the acinus regardless of magnification. Other limits for detection of prominent nucleoli when diagnosing HGPIN were used by five $(8 \%)$. The remaining respondents did not answer this question.

Number of cores involved with HGPIN was reported by $32(50 \%)$. This was less often performed by North Americans (38\%) than by others (71\%), $P=0.01$.

\section{Atypical Acinar Lesions Suspicious for but not Diagnostic of Carcinoma}

Atypical acinar lesions suspicious for, but not diagnostic of, cancer on needle biopsy or TURP were reported as ASAP, with or without a note or comment by $30(47 \%)$, as suspicious for cancer by $13(20 \%)$ and as atypia or atypical glands, with or without a note or comment by $18(28 \%)$ (Table 3$)$. Of these, two used both suspicious and atypia. ASAP was used by $55 \%$ of North Americans and $33 \%$ of others $(P=0.09)$. Other terminology was used by five $(8 \%)$. 
Table 3 Atypical acinar lesions suspicious for but not diagnostic of carcinoma

\begin{tabular}{lc} 
Question & $\begin{array}{c}\text { Percent } \\
\text { (number) of } \\
\text { respondents }\end{array}$ \\
\hline $\begin{array}{l}\text { Terminology of atypical acinar lesions suspicious } \\
\text { diagnostic of barcinoma }\end{array}$ & $47(30)$ \\
$\quad$ Atypical small acinar proliferation (ASAP) & $20(13)$ \\
Suspicious for cancer & $28(18)$ \\
Atypia/atypical glands & \\
& \\
Reporting of degree of suspicion of cancer & $41(26)$ \\
Yes & $59(38)$ \\
No & \\
Terminology for degree of suspicion of cancer & $9(6)$ \\
Favor benign, favor malignant & $8(5)$ \\
Favor benign, equivocal, favor malignant & $14(9)$ \\
Weak, intermediate, strong suspicion for & $14(9)$ \\
cancer & \\
Other & \\
Recommendation of repeat biopsy in cases with atypical glands \\
suspicious of cancer \\
Yes, always \\
Sometimes (depending on referring doctor) \\
Sometimes (depending on patient age) \\
No
\end{tabular}

Different diagnostic categories to further define the degree of suspicion of carcinoma were used by $26(41 \%)$. Categories of suspicion were used by $33 \%$ of North Americans and $54 \%$ of others $(P=0.09)$. The categories used for this were: favor benign, favor malignant used by six ( $9 \%)$, favor benign, equivocal for malignancy, favor malignant used by five (8\%) and weak suspicion for cancer, intermediate suspicion for cancer, strong suspicion for cancer used by nine $(14 \%)$. Other methods to categorize the suspicion were used by nine (14\%). Of the respondents, three used more than one method to designate categories of suspicion.

In needle biopsy cases with atypical glands suspicious for but not diagnostic of cancer, 22 $(34 \%)$ would always add a comment that repeat biopsy is recommended, $18(28 \%)$ would not, and $24(37 \%)$ would add a comment depending on whom the referring doctor is (30\%) and/or depending on age of patient $(13 \%)$. A recommendation was always issued by $43 \%$ of North Americans and by $21 \%$ of others, and never issued by $20 \%$ of North Americans and $42 \%$ of others $(P=0.10)$.

\section{Discussion}

Historically, there has been some confusion about the nomenclature for atypical lesions of the prostate. Terms such as dysplasia, duct-acinar dysplasia, atypia, atypical hyperplasia, intraductal carcinoma and carcinoma in situ have been used for atypical lesions that are thought to be noninvasive. ${ }^{6,7}$ The preneoplastic nature of nuclear atypia in secretory cells of prostatic glands or ducts was shown by McNeal et $a l^{7,8}$ They used the term duct-acinar dysplasia because the distinction between ducts and acini is arbitrary and often difficult in limited histological specimens such as needle biopsies., ${ }^{7,8}$ Some pathologists are reluctant to use the term dysplasia for preneoplastic lesions because dysplasia also refers to developmental disorders. Therefore, the acronym PIN was introduced in 1987, in accordance with CIN of the uterine cervix. ${ }^{2}$ PIN rapidly gained acceptance among GU pathologists and was uniformly endorsed by the respondents of this survey. However, $44 \%$ still used intraductal carcinoma to designate a lesion that has necrosis or much more pronounced cytological or architectural atypia than typical PIN. Criteria for distinguishing intraductal cancerization from HGPIN have been defined by McNeal and Yemoto. ${ }^{9}$ It has been postulated that intraductal carcinoma is associated with aggressive prostate carcinoma, ${ }^{10}$ but it remains controversial whether intraductal carcinoma actually can be separated from cribriform HGPIN. ${ }^{11}$

The first years after its introduction, PIN was graded according to a three-tier system. ${ }^{2,7,12}$ However, when a panel of experts in GU pathology reviewed a set of slides including biopsies with benign tissue, PIN and cancer, the reproducibility of benign lesions vs PIN 1 was poor and the same was true for PIN 2 vs $3 .^{5}$ This supported the recommendation from a consensus meeting in Bethesda 1989 that PIN 2 and 3 should be lumped together as HGPIN and PIN 1 be replaced by LGPIN. ${ }^{13}$ A recommendation was later issued that LGPIN should not be reported because of its lack of specificity. ${ }^{14}$ It seems that the vast majority of GU pathologists have adopted these recommendations, although $25 \%$ of the survey respondents would occasionally mention LGPIN in the descriptive part of the report if the lesion is prominent. Similarly, some of us would comment on LGPIN in consultation cases where a submitting diagnosis of HGPIN was considered or rendered.

Bostwick et $a l^{15}$ described four architectural patterns of HGPIN: cribriform, micropapillary, tufted and flat PIN. Several studies have shown that cribriform PIN conveys an increased risk of developing into invasive cancer compared to other types of PIN. ${ }^{16,17}$ Others have failed to confirm this finding. ${ }^{18}$ The split opinion on the prognostic impact of PIN architecture may explain that only $19 \%$ of respondents specified architectural patterns in needle biopsy reports. Cribriform PIN was only marginally more often specified than other patterns (16 vs 13\%).

There have been numerous reports that when HGPIN but no invasive cancer is found in a needle biopsy, there is an increased risk of having cancer in a subsequent biopsy. Initially, a high risk was reported, usually in the interval of $30-40 \% .^{17,19}$ 
Several recent studies have shown a somewhat lower risk at $(26-30 \%) .^{16,18,20}$ The decreased predictive value for cancer may be explained by an increasing number of cores in each biopsy session. When no cancer is found in multiple biopsy cores, the risk of missing an invasive cancer is evidently lower than when a limited number of cores are taken. Another possible explanation is that current screening programs have decreased the number of undetected invasive carcinomas in the population. Possibly due to the decreasing incidence in the reported risk of cancer following an initial diagnosis of HGPIN on biopsy, only $6 \%$ would always add a comment that HGPIN is a premalignant lesion and only $8 \%$ would always recommend a repeat biopsy.

HGPIN has significant nuclear atypia in secretory cells, while basal cells are unaffected and preserved. Therefore, immunohistochemistry for basal cell markers may help to distinguish between HGPIN and invasive carcinoma. A diagnostic problem is that the basal cell layer of HGPIN may be patchy and occasionally absent around small outpouchings of a larger PIN gland. ${ }^{21}$ Hence, for the assessment of single atypical glands where the differential diagnosis is between HGPIN and invasive carcinoma, the value of immunohistochemistry is limited and the diagnosis should mainly be based on routine stained sections. An overwhelming majority (92\%) consequently would use immunohistochemistry in less than $20 \%$ of HGPIN cases.

By definition, HGPIN has prominent nucleoli in addition to general nuclear atypia with anisokaryosis, hyperchromasia and nuclear enlargement and crowding. ${ }^{5,7}$ The definition of prominent nucleoli is unclear and subjective. Pathologists cannot be expected to measure nucleolar diameter in their daily practice. In the survey, we defined nucleolar enlargement according to the magnification at which they could be detected. Even so, there was a wide variation between respondents in terms of what is required for a nucleolus to be considered enlarged. Moreover, the number of prominent nucleoli needed for a diagnosis of HGPIN was never defined when PIN was introduced. ${ }^{2,7}$ Nevertheless, as many as $33 \%$ would require prominent nucleoli in at least $10 \%$ of secretory cells to diagnose HGPIN. In summary, the definition of HGPIN needs to be standardized both with regard to the definition of nucleolar prominence and the number of prominent nucleoli.

Especially in consultation practice, the quality of sections and stainings may be unsatisfactory. In thick or overstained sections prominent nucleoli are sometimes difficult to observe. Yet, glands may display such a pronounced atypia that a diagnosis of HGPIN is suggested. Despite the emphasis on nucleolar size in the definition of HGPIN, $56 \%$ of respondents would diagnose HGPIN without prominent nucleoli, if there was a sufficient atypia with either prominent pleomorphism, marked hyperchro- masia, mitotic figures, micropapillary pattern, cribriform pattern or extensive lesion.

Several studies have found that the number of cores involved with HGPIN is predictive for cancer in subsequent biopsies. ${ }^{16,17}$ In contrast, others have failed to demonstrate an increased risk of cancer with multiple cores involved. ${ }^{18,22}$ The number of cores with HGPIN was only reported by $50 \%$ of survey respondents reflecting the conflicting data on this issue.

Lesions suspicious for but not diagnostic of carcinoma were reported as atypia, atypical glands or suspicious for cancer by $45 \%$ and as ASAP by $42 \%$. The term ASAP was introduced in $1993^{3}$ and has been debated since then. ${ }^{23}$ There are a number of disadvantages with the use of ASAP. Not all lesions suspicious for cancer have small glands and these lesions are occasionally not even acinar. The acronym ASAP may also suggest that this is a diagnostic entity, rather than an expression of the degree of diagnostic uncertainty. In a recent survey on how urologists interpret pathology reports on prostate biopsies, it appeared that $98 \%$ of respondents would rebiopsy a patient with ASAP on initial biopsy. ${ }^{24}$ However, only $29 \%$ of urologists thought of ASAP as more alarming than HGPIN. Despite reports that ASAP predicts cancer in $29-75 \%$ of cases (mean 43\%), ${ }^{25}$ some clinicians seem to be unaware of the magnitude of this risk. We believe that underestimation of the risk of cancer can be avoided by adding a statement to the report that biopsy findings are suspicious for malignancy although insufficient for a conclusive diagnosis of cancer. In line with this, the 2004 World Health Organization-sponsored International Consultation issued a recommendation to designate atypical biopsies as either suspicious or highly suspicious for cancer, rather than using the diagnosis ASAP without a comment. ${ }^{26}$

In summary, there was a reasonable agreement on the terminology of preneoplastic lesions, grading of PIN and the lack of clinical significance of LGPIN. However, there is a need of standardization of diagnostic criteria for HGPIN and intraductal carcinoma and of the reporting of number of cores involved with HGPIN.

\section{Acknowledgements}

We thank the following pathologists for responding to the questionnaire: Ferran Algaba, Mahul Amin, Sheldon Bastacky, Aasmund Berner, Athanase Billis, Liliane Boccon-Gibod, Christer Busch, Liang Cheng, John Cheville, Stephen Cina, Francisco Civantos, Cynthia Cohen, Charles Davis, Brett Delahunt, Warick Delprado, Anthony di Sant'Agnese, John Eble, Fang Fan, Masakuni Furusato, Paul Gaudin, Neal Goldstein, Hans Hamberg, Burkhardt Helpap, Peter Humphrey, Kenneth Iczkowski, Sonny Johansson, Edward Jones, Hillel Kahane, Theo van 
der Kwast, Howard Levin, Agnes Kathrine Lie, Antonio López Beltrán, Gregor Mikuz, Rodolfo Montironi, Robin Moseley, Ray Nagle, Lucien Nochomovitz, Stig Nordling, Gerard O'Dowd, Roberto Orozco, Kathleen O’Toole, Chin-Chen Pan, Carl-Gustaf Pihl, Victor Reuter, Mark Rubin, Hemamali Samaratunga, Thomas Sebo, Isabell Sesterhenn, Maria Shevchuk, John R Srigley, Puay Hoon Tan, Bernard Têtu, John Tomaszewski, Patricia Troncoso, Lawrence True, Toyonori Tsuzuki, Thomas Ulbright, Thomas Wheeler, Kirk Wojno, Ximing Yang and Robert Young.

\section{References}

1 Egevad L, Allsbrook Jr WC, Epstein JI. Current practice of Gleason grading among genitourinary pathologists. Hum Pathol 2005;36:5-9.

2 Bostwick DG, Brawer MK. Prostatic intra-epithelial neoplasia and early invasion in prostate cancer. Cancer 1987;59:788-794.

3 Bostwick DG, Srigley J, Grignon D, et al. Atypical adenomatous hyperplasia of the prostate: morphologic criteria for its distinction from well-differentiated carcinoma. Hum Pathol 1993;24:819-832.

4 Iczkowski KA, MacLennan GT, Bostwick DG. Atypical small acinar proliferation suspicious for malignancy in prostate needle biopsies: clinical significance in 33 cases. Am J Surg Pathol 1997;21:1489-1495.

5 Epstein JI, Grignon DJ, Humphrey PA, et al. Interobserver reproducibility in the diagnosis of prostatic intraepithelial neoplasia. Am J Surg Pathol 1995;19:873-886.

6 Kastendieck H, Helpap B. Prostatic 'dysplasia/atypical hyperplasia’. Terminology, histopathology, pathobiology, and significance. Urology 1989;34:28-42.

7 McNeal JE. Significance of duct-acinar dysplasia in prostatic carcinogenesis. Prostate 1988;13:91-102.

8 McNeal JE. Morphogenesis of prostatic carcinoma. Cancer 1965;18:1659-1666.

9 McNeal JE, Yemoto CE. Spread of adenocarcinoma within prostatic ducts and acini. Morphologic and clinical correlations. Am J Surg Pathol 1996;20:802-814.

10 Cohen RJ, McNeal JE, Baillie T. Patterns of differentiation and proliferation in intraductal carcinoma of the prostate: significance for cancer progression. Prostate 2000;43:11-19.

11 Rubin MA, de La Taille A, Bagiella E, et al. Cribriform carcinoma of the prostate and cribriform prostatic intraepithelial neoplasia: incidence and clinical implications. Am J Surg Pathol 1998;22:840-848.

12 McNeal JE, Bostwick DG. Intraductal dysplasia: a premalignant lesion of the prostate. Hum Pathol 1986; 17:64-71.

13 Troncoso P, Babaian RJ, Ro JY, et al. Prostatic intraepithelial neoplasia and invasive prostatic adeno- carcinoma in cystoprostatectomy specimens. Urology 1989;34:52-56.

14 Srigley JR, Amin MB, Bostwick DG, et al. Updated protocol for the examination of specimens from patients with carcinomas of the prostate gland: a basis for checklists. Cancer Committee. Arch Pathol Lab Med 2000;124:1034-1039.

15 Bostwick DG, Amin MB, Dundore P, et al. Architectural patterns of high-grade prostatic intraepithelial neoplasia. Hum Pathol 1993;24:298-310.

16 Bishara T, Ramnani DM, Epstein JI. High-grade prostatic intraepithelial neoplasia on needle biopsy: risk of cancer on repeat biopsy related to number of involved cores and morphologic pattern. Am J Surg Pathol 2004;28:629-633.

17 Kronz JD, Allan CH, Shaikh AA, et al. Predicting cancer following a diagnosis of high-grade prostatic intraepithelial neoplasia on needle biopsy: data on men with more than one follow-up biopsy. Am J Surg Pathol 2001;25:1079-1085.

18 Gokden N, Roehl KA, Catalona WJ, et al. High-grade prostatic intraepithelial neoplasia in needle biopsy as risk factor for detection of adenocarcinoma: current level of risk in screening population. Urology 2005; 65:538-542.

19 Davidson D, Bostwick DG, Qian J, et al. Prostatic intraepithelial neoplasia is a risk factor for adenocarcinoma: predictive accuracy in needle biopsies. J Urol 1995;154:1295-1299.

20 Lefkowitz GK, Taneja SS, Brown J, et al. Followup interval prostate biopsy 3 years after diagnosis of high grade prostatic intraepithelial neoplasia is associated with high likelihood of prostate cancer, independent of change in prostate specific antigen levels. J Urol 2002;168:1415-1418.

21 Kronz JD, Shaikh AA, Epstein JI. High-grade prostatic intraepithelial neoplasia with adjacent small atypical glands on prostate biopsy. Hum Pathol 2001;32: 389-395.

22 Naya Y, Ayala AG, Tamboli P, et al. Can the number of cores with high-grade prostate intraepithelial neoplasia predict cancer in men who undergo repeat biopsy? Urology 2004;63:503-508.

23 Epstein JI. How should atypical prostate needle biopsies be reported? Controversies regarding the term 'ASAP'. Hum Pathol 1999;30:1401-1402.

24 Rubin MA, Bismar TA, Curtis S, et al. Prostate needle biopsy reporting: how are the surgical members of the Society of Urologic Oncology using pathology reports to guide treatment of prostate cancer patients? Am J Surg Pathol 2004;28:946-952.

25 Humphrey PA. Prostate Pathology. ASCP Press: Chicago, 2003.

26 Amin M, Boccon-Gibod L, Egevad L, et al. Prognostic and predictive factors and reporting of prostate carcinoma in prostate needle biopsy specimens. Scand J Urol Nephrol Suppl 2005;216:20-33. 